\title{
Scrutinizing the Average Error Probability for Nakagami Fading Channels
}

\author{
Gholamreza Alirezaei \\ Inst. for Theoretical Information Technology \\ RWTH Aachen University \\ D-52056 Aachen, Germany \\ alirezaei@ti.rwth-aachen.de
}

\author{
Rudolf Mathar \\ Inst. for Theoretical Information Technology \\ RWTH Aachen University \\ D-52056 Aachen, Germany \\ mathar@ti.rwth-aachen.de
}

\begin{abstract}
The ultimate goal of the present paper is to provide mathematical tools for dealing with the complicated average error probability (AEP) in Nakagami fading channels. This is useful for analytical investigations as well as alleviating computational effort in simulations or on-line computations. We hence thoroughly analyze the mathematical structure of the AEP over Nakagami fading channels. First, the AEP is re-parameterized to obtain a mathematically concise form. The main contributions are then as follows. An ordinary differential equation is set up, which has the AEP as a solution. By this approach, a new representation of the AEP is found, which merely needs integration over a broken rational function. This paves the way to numerous amazing relations of the AEP, e.g., to the Gaussian hypergeometric and the incomplete beta function. Moreover, monotonicity and logconvexity are demonstrated. Finally, asymptotic expansions of the AEP are given.
\end{abstract}

\section{INTRODUCTION}

Understanding the stochastic nature of fast fading channels is a prerequisite to develop high performance transceivers. Additive white Gaussian noise channels are well investigated, however, practically relevant channels like Rice and Nakagami fading channels are much less understood. The main reason is that analytical expressions become mathematically intractable because highly complicated functions are involved. Hence, in simulations only numerical methods are used to optimize, analyze or verify the performance of communication links. These methods are indeed useful for many applications, but inadequate to truly understand and describe the behavior of transceivers and their performance.

A prominent example is the explicit evaluation of the average error probability (AEP) when transmitting over Rice or Nakagami fading channels. A closed-form expression of the average error probability from its integral representation seems barely achievable except in some rare special cases. In this situation, mathematical approximations by simpler functions are of great help and fully sufficient for most practical purposes.

It is the main purpose of the present paper to provide a bunch of mathematical tools for dealing with the complicated AEP in Nakagami fading channels. This is useful for analytical investigations as well as alleviating the computational effort in simulations or on-line computations.

In this paper, we explore the AEP over Nakagami fading channels in detail. The present work may be seen in the same vein as the pioneering works [1]-[6] and [7]. Therein the error probability and corresponding approximations in terms of special functions and finite or infinite series are investigated. In [8] characteristic functions are used to show that the average error probability analysis of different modulation formats can be unified into a single common framework.

In the next section, we start with the prerequisite mathematical material. Thereafter, the AEP is represented as an integral and re-parameterized to obtain a mathematically concise form. The next step consists of setting up an ordinary differential equation, which has AEP as a solution. By this approach, a convenient representation is found, which merely needs integration over a broken rational function. This paves the way to numerous amazing relations of the AEP, e.g., to the Gaussian hypergeometric and the incomplete beta function. Moreover, monotonicity and log-convexity are demonstrated. Finally, asymptotic expansions of the AEP are given. In summary, a set of analytical tools is provided which supports numerical computations on one hand and allows for developing simple approximations and bounds of the error probability on the other hand.

\section{Mathematical Preliminaries}

The sets of positive (non-negative) integers and (nonnegative) real numbers are denoted by by $\mathbb{N}\left(\mathbb{N}_{0}\right)$ and $\left(\mathbb{R}_{+}\right)$ $\mathbb{R}$, respectively. By $|z|$ we denote the absolute value of some real number $z$. We further introduce some important special functions and summarize their properties, which may be found for example in chapters 6, 7, 15 and 26 of [9].

Euler's classical gamma function is defined as

$$
\Gamma(x):=\int_{0}^{\infty} t^{x-1} \mathrm{e}^{-t} \mathrm{~d} t, \quad x>0 .
$$

It is well-known that

$$
\Gamma\left(\frac{1}{2}\right)=\sqrt{\pi}, \Gamma(1)=\Gamma(2)=1 \text { and } \Gamma\left(\frac{3}{2}\right)=\frac{\sqrt{\pi}}{2} .
$$

Moreover, for all positive real numbers $x$, the identity

$$
\Gamma(x+n)=\Gamma(x) \prod_{i=0}^{n-1}(x+i), \quad n \in \mathbb{N},
$$


holds, which especially entails $\Gamma(x+1)=x \Gamma(x)$. Furthermore, it holds that

$$
\lim _{n \mapsto \infty} n^{b-a} \frac{\Gamma(n+a)}{\Gamma(n+b)}=1, \quad a, b \in \mathbb{R}_{+} .
$$

Closely related to the gamma function is the incomplete beta function, which is defined as

$$
\mathrm{B}(a, b ; x):=\int_{0}^{x} t^{a-1}(1-t)^{b-1} \mathrm{~d} t
$$

for all $a, b>0$ and $0 \leq x \leq 1$. By substituting $t$ by $\frac{t}{1+t}$ in (5), we obtain the identity

$$
\mathrm{B}(a, b ; x)=\int_{0}^{\frac{x}{1-x}} \frac{t^{a-1}}{(1+t)^{a+b}} \mathrm{~d} t
$$

The beta function is obtained as $x \rightarrow 1$ yielding

$$
\mathrm{B}(a, b):=\frac{\Gamma(a) \Gamma(b)}{\Gamma(a+b)}=\mathrm{B}(b, a)=\int_{0}^{\infty} \frac{t^{a-1}}{(1+t)^{a+b}} \mathrm{~d} t .
$$

The Gaussian error function and a useful series expansion are given for $x \in \mathbb{R}$ by

$$
\operatorname{erfc}(x):=\frac{2}{\sqrt{\pi}} \int_{x}^{\infty} \mathrm{e}^{-t^{2}} \mathrm{~d} t=1-\frac{2}{\sqrt{\pi}} \sum_{n=0}^{\infty} \frac{(-1)^{n} x^{2 n+1}}{(2 n+1) n !} .
$$

Another special function of importance for this work is the Gaussian hypergeometric function. For all $a, b$ and $c \in \mathbb{R}_{+}$ and for all $x \in \mathbb{R}$, it is defined as

${ }_{2} \mathrm{~F}_{1}(a, b ; c ; x):=\frac{\Gamma(c)}{\Gamma(a) \Gamma(b)} \sum_{n=0}^{\infty} \frac{\Gamma(a+n) \Gamma(b+n) x^{n}}{\Gamma(c+n) \Gamma(n+1)}$.

If $a, b>0$ and $c>b$, then for all $x>0$ the integral representation

$$
{ }_{2} \mathrm{~F}_{1}(a, b ; c ;-x)=\frac{\Gamma(c)}{\Gamma(b) \Gamma(c-b)} \int_{0}^{1} \frac{t^{b-1}(1-t)^{c-b-1}}{(1+x t)^{a}} \mathrm{~d} t
$$

holds.

Definition 1 For all real numbers $x>-2$, we define the ratio

$$
\tau(x):=\frac{\sqrt{\pi} \Gamma\left(\frac{x+3}{2}\right)}{\Gamma\left(\frac{x+2}{2}\right)} .
$$

Obviously, $\tau(x)$ is positive. Furthermore, the identities

$$
\tau(-1)=1, \tau(0)=\frac{\pi}{2} \text { and } \tau(1)=2
$$

easily follow from (2). From (3) and (7) we conclude that

$$
\tau(x)=\frac{x+1}{2} \int_{0}^{\infty} \frac{\mathrm{d} t}{(1+t)^{\frac{x+2}{2}} \sqrt{t}} .
$$

\section{Mathematical Structure of the NaKagami ERROR PROBABILITY}

For many concrete modulation schemes the bit and symbol error probability is described by the function

$$
P(\xi):=c_{1} \operatorname{erfc}\left(c_{2} \sqrt{\xi}\right) .
$$

Constants $c_{1}$ and $c_{2}$ depend on the modulation scheme, and variable $\xi$ denotes the signal-to-noise ratio (SNR) at the receiver.

Coherently detected M-ary PAM [10, p. 265] and BPSK [10, p. 268] are examples where the error probability is exactly given by equation (14). The error probability of M-ary PSK, M-ary QAM [11, p. 180], and M-ary FSK [12, p. 230] can be accurately approximated by formula (14).

We now assume that the SNR is a random variable and the envelope of the channel response is Nakagami- $m$ distributed see [13]. Then the average error probability (AEP) is given by

$$
P_{\text {avg }}(m, \bar{\xi}):=\int_{0}^{\infty} P(s)\left(\frac{m}{\bar{\xi}}\right)^{m} \frac{s^{m-1}}{\Gamma(m)} \exp \left(-\frac{m s}{\bar{\xi}}\right) \mathrm{d} s,
$$

where $\bar{\xi}$ denotes the expected value of $\xi$ and parameter $m$ is called fading-figure, see [14].

Applying the parameter transformations

$$
m=\frac{p+1}{2}, \bar{\xi}=\frac{p+1}{2 x c_{2}^{2}} \text { and } s=\frac{t^{2}}{c_{2}^{2}},
$$

and multiplying by $c_{1}^{-1} \tau(p) x^{-\frac{p+1}{2}}$ leads to a concise form of the integral which is central to what follows and is highlighted as a definition.

Definition 2 For all $p \in \mathbb{R},-1<p<\infty$ and for all $x \in \mathbb{R}_{+}$, we give the name Beta-Nakagami Integral (BeNaI) to

$$
g(p, x):=\frac{(p+1) \sqrt{\pi}}{\Gamma\left(\frac{p+2}{2}\right)} \int_{0}^{\infty} t^{p} \mathrm{e}^{-x t^{2}} \operatorname{erfc}(t) \mathrm{d} t .
$$

This name will later become clear from property (29c).

Note that $g(p, 0)=1$, which results from the identity ${ }^{1}$

$$
\int_{0}^{\infty} t^{p} \operatorname{erfc}(t) \mathrm{d} t=\frac{\Gamma\left(\frac{p+2}{2}\right)}{(p+1) \sqrt{\pi}},
$$

see $[15$, p. 56, eq. 12].

The explicit relation between (15) and (17) reads as follows

$$
P_{\text {avg }}\left(\frac{p+1}{2}, \frac{p+1}{2 x c_{2}^{2}}\right)=\frac{x^{\frac{p+1}{2}} c_{1}}{\tau(p)} g(p, x) .
$$

In applications, the AEP and BeNaI are usually numerically evaluated. In order to decrease computational effort and to improve numerical accuracy, new identities are derived in the following. We start by determining the derivatives of $g(p, x)$.

\footnotetext{
${ }^{1}$ Identity (18) is well-known and was first published in [15, p. 56, eq. 12].
} 
Lemma 3 For all $n \in \mathbb{N}$, the $n^{\text {th }}$ partial derivative of $g(p, x)$ with respect to $x$ is given by

$$
\begin{aligned}
g^{(n)}(p, x) & :=\frac{\partial^{n} g(p, x)}{\partial x^{n}} \\
& =(-1)^{n} \frac{p+1}{p+1+2 n} g(p+2 n, x) \prod_{m=1}^{n} \frac{p+2 m}{2} .
\end{aligned}
$$

In particular,

$$
g^{\prime}(p, x):=\frac{\partial g(p, x)}{\partial x}=-\frac{p+1}{2} \frac{p+2}{p+3} g(p+2, x) .
$$

Proof: Differentiating $g(p, x) n$-times with respect to $x$ and using (3) gives

$$
\begin{aligned}
g^{(n)}(p, x) & =(-1)^{n} \frac{(p+1) \sqrt{\pi}}{\Gamma\left(\frac{p+2}{2}\right)} \int_{0}^{\infty} t^{p+2 n} \mathrm{e}^{-x t^{2}} \operatorname{erfc}(t) \mathrm{d} t \\
& =(-1)^{n} \frac{\Gamma\left(\frac{p+2}{2}+n\right)}{\Gamma\left(\frac{p+2}{2}\right)} \frac{p+1}{p+1+2 n} g(p+2 n, x) \\
& =(-1)^{n} \frac{p+1}{p+1+2 n} g(p+2 n, x) \prod_{m=1}^{n} \frac{p+2 m}{2}
\end{aligned}
$$

Lemma 4 The BeNaI satisfies the following ordinary differential equation

$$
g(p, x)+\frac{2 x}{p+1} g^{\prime}(p, x)=\frac{1}{(1+x)^{\frac{p+2}{2}}} .
$$

Proof: Considering (17) and using integration by parts, where $t^{p}$ is integrated and $\mathrm{e}^{-x t^{2}} \operatorname{erfc}(t)$ is differentiated with respect to $t$, we deduce the identity

$$
g(p, x)=\frac{p+2}{p+3} x g(p+2, x)+\frac{1}{(1+x)^{\frac{p+2}{2}}} .
$$

Using (21) yields the result (23).

Theorem 5 For all $-1<p<\infty$ and for all $x \in \mathbb{R}_{+}$, the BeNaI has the series representation

$$
g(p, x)=\frac{p+1}{p(1+x)^{\frac{p+2}{2}}} \sum_{n=0}^{\infty}\left(\frac{x}{1+x}\right)^{n} \prod_{m=0}^{n} \frac{p+2 m}{p+1+2 m} .
$$

Note that $p=0$ is a removable discontinuity (singularity).

Proof: The proof consists of showing that the series representation (25) satisfies the differential equation (23). By

\footnotetext{
${ }^{2}$ Since $g(p, x)$ has roots to the beta-prime distribution and arises from the Nakagami distribution, we call it Beta-Nakagami Integral (BeNaI), as stated in Definition 2.
}

elementary algebra we obtain

$$
\begin{aligned}
\frac{p+2}{p+3} & x g(p+2, x) \\
& =\frac{x}{(1+x)^{\frac{p+4}{2}}} \sum_{n=0}^{\infty}\left(\frac{x}{1+x}\right)^{n} \prod_{m=0}^{n} \frac{p+2+2 m}{p+3+2 m} \\
& =\frac{1}{(1+x)^{\frac{p+2}{2}}} \sum_{i=1}^{\infty}\left(\frac{x}{1+x}\right)^{i} \prod_{m=0}^{i-1} \frac{p+2+2 m}{p+3+2 m} \\
& =\frac{p+1}{p(1+x)^{\frac{p+2}{2}}} \sum_{i=1}^{\infty}\left(\frac{x}{1+x}\right)^{i} \prod_{j=0}^{i} \frac{p+2 j}{p+1+2 j} \\
& =g(p, x)-\frac{1}{(1+x)^{\frac{p+2}{2}}},
\end{aligned}
$$

which is equivalent to (24).

Theorem 6 It holds that

$$
\begin{aligned}
g(p, x) & =\frac{p+1}{2 x^{\frac{p+1}{2}}} \int_{0}^{x} \frac{t^{\frac{p-1}{2}}}{(1+t)^{\frac{p+2}{2}}} \mathrm{~d} t \\
& =\frac{p+1}{2} \int_{0}^{1} \frac{t^{\frac{p-1}{2}}}{(1+x t)^{\frac{p+2}{2}}} \mathrm{~d} t
\end{aligned}
$$

for all $-1<p<\infty$ and for all $x \in \mathbb{R}_{+}$.

Proof: The proof is by inspection. The right hand side of (27a) is a solution of differential equation (23) as can be seen from

$$
\begin{gathered}
\frac{\partial}{\partial x}\left[\frac{p+1}{2 x^{\frac{p+1}{2}}} \int_{0}^{x} \frac{t^{\frac{p-1}{2}}}{(1+t)^{\frac{p+2}{2}}} \mathrm{~d} t\right] \\
=\frac{p+1}{2}\left[-\frac{p+1}{2 x^{\frac{p+3}{2}}} \int_{0}^{x} \frac{t^{\frac{p-1}{2}}}{(1+t)^{\frac{p+2}{2}}} \mathrm{~d} t+\frac{1}{x^{\frac{p+1}{2}}} \frac{x^{\frac{p-1}{2}}}{(1+x)^{\frac{p+2}{2}}}\right] \\
=\frac{p+1}{2 x}\left[-g(p, x)+\frac{1}{(1+x)^{\frac{p+2}{2}}}\right] .
\end{gathered}
$$

Since the initial values of (17) and (27a) are equal, both representations coincide.

Representation (27b) is obtained by substituting $t$ by $t x$ in equation (27a).

We are now in a position to prove some interesting connections between the $\mathrm{BeNaI}$ and the special functions ${ }_{2} \mathrm{~F}_{1}(\cdot)$ and $\mathrm{B}(\cdot)$ from (9) and (5), respectively.

\section{Corollary 7 It holds that ${ }^{2}$}

$$
\begin{aligned}
g(p, x) & ={ }_{2} \mathrm{~F}_{1}\left(\frac{p+1}{2}, \frac{p+2}{2} ; \frac{p+3}{2} ;-x\right) \\
& =\frac{1}{(1+x)^{\frac{p}{2}}}{ }_{2} \mathrm{~F}_{1}\left(\frac{1}{2}, 1 ; \frac{p+3}{2} ;-x\right) \\
& =\frac{p+1}{2 x^{\frac{p+1}{2}}} \mathrm{~B}\left(\frac{p+1}{2}, \frac{1}{2} ; \frac{x}{1+x}\right)
\end{aligned}
$$

for all $-1<p<\infty$ and for all $x \in \mathbb{R}_{+}$. 
Proof: By using integral identity (10), representation (27b) and equation (3) we conclude (29a), see also equation (20) in [16, p. 310]. By applying the transformation formula [9, p. 559, eq. 15.3.3], we obtain identity (29b). Equation (29c) follows from (6) and (27a).

A short verification: In order to verify the distribution of the SNR, we start the following discussion from an alternative representation of the BeNaI. Because of identity (29c) the $\mathrm{BeNaI}$ is related to the cumulative density function (CDF) of a real positive random variable which has a beta prime distribution or synonymously a beta distribution of the second kind, see [17, p. 50, eq. 4.3] and [18, p. 248, eq. 25.79]. The corresponding CDF of a beta prime distributed random variable $X$ with parameters $a$ and $b$ is in general described by $\operatorname{Prob}(X \leq x)=\frac{1}{\mathrm{~B}(a, b)} \mathrm{B}\left(a, b ; \frac{x}{1+x}\right)$. The random variable $X=\frac{X_{1}}{X_{2}}$ is beta prime distributed with parameters $a=\frac{p+1}{2}$ and $b=\frac{1}{2}$, if both random variables $X_{1}$ and $X_{2}$ are independent and gamma distributed with parameters $k_{1}=p+1$ and $k_{2}=1$, respectively. Note that we only consider a particular case of gamma distributions for which the density is defined as $\frac{x^{k-1} \mathrm{e}^{-x / 2}}{\Gamma(k) 2^{k}}$ with parameter $k>0$. In turn, the random variables $X_{1}=Y_{1}^{2}$ and $X_{2}=Y_{2}^{2}$ are gamma distributed, if the random variables $Y_{1}$ and $Y_{2}$ are Nakagami and standard normal distributed, respectively. For example, the random variable $Y_{2}$ could describe the AWGN at the receiver, while the variable $Y_{1}$ might model the underlying multi-path propagation channel. This short verification confirms the validity of the underlaying communication model and the distribution of the SNR, which is discussed in the introduction of this section.

By applying transformations from [9] and [19], other identities are accessible by analogous methods. In particular, relations to the associated Legendre function of the second kind with complex argument and to Meijer's G-function can be achieved.

The new identities (27a) and (27b) facilitate numerical computations of $g(p, x)$ for certain $p$ and $x$ significantly compared to direct use of representation (17). There are three reasons for this fact. First, the integration domain is finite. The integrand includes only a singularity, if $-1<p<1$ holds. Second, the integrand does not contain any complicated functions, and third, all operations and functions in the integrand are numerically stable.

Simplicity and stability hold for computing the AEP from (27a), as well. This can be seen by incorporating (3), (13) and (27a) into (19), which gives ${ }^{3}$

$$
\begin{aligned}
P_{\text {avg }}\left(\frac{p+1}{2},\right. & \left.\frac{p+1}{2 x c_{2}^{2}}\right)=\frac{c_{1}}{\pi} \tau(p-1) \int_{0}^{x} \frac{t^{\frac{p-1}{2}} \mathrm{~d} t}{(1+t)^{\frac{p+2}{2}}} \\
= & \frac{p c_{1}}{2 \pi} \int_{0}^{\infty} \frac{\mathrm{d} t}{(1+t)^{\frac{p+1}{2}} \sqrt{t}} \int_{0}^{x} \frac{t^{\frac{p-1}{2}} \mathrm{~d} t}{(1+t)^{\frac{p+2}{2}}} .
\end{aligned}
$$

\footnotetext{
${ }^{3} \mathrm{~A}$ transformed version of identity (30) in terms of regularized incomplete beta function is already discussed in [6, eq. A.5]. However, its solution is given without a full proof.
}

Furthermore, by the new representations, it becomes much easier to calculate the BeNaI explicitly in certain special cases, as is demonstrated by the following examples.

Example 8 For $p=1$ and all $x \geq 0$ it holds that ${ }^{4}$

$$
g(1, x)=\frac{2}{1+x+\sqrt{1+x}} .
$$

Equation (31) follows from Theorem 6 by substituting $t$ by $t-1$ and integrating.

Example 9 For $p=0$ and all $x \geq 0$ it holds that ${ }^{5}$

$$
g(0, x)=\frac{1}{\sqrt{x}} \arctan (\sqrt{x})
$$

The assertion follows from Theorem 6 by substituting $t$ by $t^{2}$.

Example 10 For $p=-1$ and all $x \geq 0$ it holds that

$$
\lim _{p \mapsto-1} g(p, x)=1 .
$$

Proof: The limit of $g(p, x)$ as $p$ approaches -1 is not obvious when starting from equation (17). However, this limit follows easily from (24) by using (31).

\section{Functional Properties of the BeNaI}

In this section, we investigate the behavior of the BeNaI (17) as a function of argument $x$, particularly its monotonicity and convexity. Moreover, the limiting behavior of the BeNaI is discussed as $x \mapsto 0$ and $x \mapsto \infty$.

Positivity, continuity and monotonicity can be easily concluded from representation (17).

Lemma 11 For all $-1<p<\infty$ and for all $x \geq 0$, the function $g(p, x)$ is
a) non-negative,
b) continuous in both arguments, and
c) strictly decreasing with respect to $x$.

As is well-known, logarithmically convex functions are also convex. We hence set out to prove this stronger property.

Lemma 12 For all $-1<p<\infty$ and for all $x \geq 0, g(p, x)$ is a logarithmically convex function of argument $x$.

Proof: Log-convexity follows if

$$
g\left(p, \lambda x_{1}+(1-\lambda) x_{2}\right) \leq g^{\lambda}\left(p, x_{1}\right) g^{1-\lambda}\left(p, x_{2}\right)
$$

\footnotetext{
${ }^{4}$ Equation (31) was initially mentioned in [15, p. 49, eq. 14$]$ and derived from (17) by a certain relation to the complementary gamma function.

${ }^{5}$ Equation (32) was initially derived in [20, p. 37] using (17) and the Laplace transform.
} 
holds for all $-1<p<\infty, x_{1} \geq 0, x_{2} \geq 0$ and $1 \geq \lambda \geq 0$. By using Hölder's inequality for integrals [21, p. 140, eq. 6.9.1], we conclude from Definition 2 that

$$
\begin{aligned}
& \int_{0}^{\infty} t^{p} \mathrm{e}^{-\left(\lambda x_{1}+(1-\lambda) x_{2}\right) t^{2}} \operatorname{erfc}(t) \mathrm{d} t \\
& =\int_{0}^{\infty}\left(t^{p} \mathrm{e}^{-x_{1} t^{2}} \operatorname{erfc}(t)\right)^{\lambda}\left(t^{p} \mathrm{e}^{-x_{2} t^{2}} \operatorname{erfc}(t)\right)^{1-\lambda} \mathrm{d} t \\
& \leq\left(\int_{0}^{\infty} t^{p} \mathrm{e}^{-x_{1} t^{2}} \operatorname{erfc}(t) \mathrm{d} t\right)^{\lambda}\left(\int_{0}^{\infty} t^{p} \mathrm{e}^{-x_{2} t^{2}} \operatorname{erfc}(t) \mathrm{d} t\right)^{1-\lambda},
\end{aligned}
$$

which completes the proof.

Lemma 13 The Taylor expansion of $g(p, x)$ at $x=0$ is given by

$$
g(p, x)=\frac{p+1}{p} \sum_{n=0}^{\infty} \frac{(-1)^{n} x^{n}}{(p+1+2 n) n ! 2^{n}} \prod_{m=0}^{n}(p+2 m) .
$$

Proof: The derivatives from Lemma 3 and the fact that $g(p, 0)=1$ for all $-1<p<\infty$, see (18), entail Taylor expansion (36).

Moreover, combining (9) and (29b) gives the series expansion

$$
g(p, x)=\frac{1}{(1+x)^{\frac{p}{2}}} \frac{\Gamma\left(\frac{p+3}{2}\right)}{\sqrt{\pi}} \sum_{n=0}^{\infty} \frac{\Gamma\left(n+\frac{1}{2}\right)(-1)^{n} x^{n}}{\Gamma\left(n+\frac{p+3}{2}\right)},
$$

which is of interest in its own.

We next consider an asymptotic series expansion of $g(p, x)$ as $x \mapsto \infty$. For a definition see [22, p.11, Definition 1.3.3].

Lemma 14 The asymptotic series expansion of $g(p, x)$ as $x \mapsto \infty$ is given by

$g(p, x)=\frac{\tau(p)}{x^{\frac{p+1}{2}}}-\frac{p+1}{p x^{\frac{p+2}{2}}} \sum_{n=0}^{\infty} \frac{(-1)^{n}}{(2 n+1) n ! 2^{n} x^{n}} \prod_{m=0}^{n}(p+2 m)$.

Proof: By utilizing the series expansion of the complementary error function (8) in (17) and using the identity

$$
\frac{\Gamma\left(\frac{p+1}{2}\right)}{2 x^{\frac{p+1}{2}}}=\int_{0}^{\infty} t^{p} \mathrm{e}^{-x t^{2}} \mathrm{~d} t, p>-1, x>0,
$$

which can be derived from equation (1), it follows that

$$
\begin{aligned}
g(p, x)= & \frac{p+1}{2} \frac{\Gamma\left(\frac{p+1}{2}\right) \sqrt{\pi}}{\Gamma\left(\frac{p+2}{2}\right) x^{\frac{p+1}{2}}} \\
& -\frac{p+1}{\Gamma\left(\frac{p+2}{2}\right)} \sum_{n=0}^{\infty} \frac{(-1)^{n}}{(2 n+1) n !} \frac{\Gamma\left(\frac{p+2}{2}+n\right)}{x^{\frac{p+2}{2}+n}} .
\end{aligned}
$$

In the light of equation (3) and (11), Lemma 14 follows after some algebra.

\section{CONCLUSIONS}

Nakagami fading is a well established model of high mathematical complexity. This paper provides insight into the structure of the Nakagami error probability for various modulation schemes. Results include concise integral representations, which facilitate numerical computation and demonstrate interrelations to the incomplete beta and Gaussian hypergeometric function. Future work will be devoted to developing sharp bounds on the average error probability by even simpler parametric functions, with the aim of optimizing transceiver algorithms for Nakagami-distributed fading channels.

\section{REFERENCES}

[1] W. Lindsey, "Error probabilities for Rician fading multichannel reception of binary and n-ary signals," Information Theory, IEEE Transactions on, vol. 10, no. 4, pp. 339-350, 1964.

[2] R. Esposito, "Error probabilities for the Nakagami channel (corresp.)," Information Theory, IEEE Transactions on, vol. 13, no. 1, pp. 145-148, 1967.

[3] Y. Miyagaki, N. Morinaga, and T. Namekawa, "Error probability characteristics for CPSK signal through m-distributed fading channel," Communications, IEEE Transactions on, vol. 26, no. 1, pp. 88-100, 1978.

[4] U. Charash, "Reception through Nakagami fading multipath channels with random delays," Communications, IEEE Transactions on, vol. 27, no. 4, pp. 657-670, 1979.

[5] E. K. Al-Hussaini and A. Al-Bassiouni, "Performance of MRC diversity systems for the detection of signals with Nakagami fading," Communications, IEEE Transactions on, vol. 33, no. 12, pp. 1315-1319, 1985.

[6] A. Wojnar, "Unknown bounds on performance in Nakagami channels," Communications, IEEE Transactions on, vol. 34, no. 1, pp. 22-24, 1986.

[7] N. Beaulieu and A. Abu-Dayya, "Analysis of equal gain diversity on Nakagami fading channels," Communications, IEEE Transactions on, vol. 39, no. 2, pp. 225-234, 1991.

[8] A. Annamalai, C. Tellambura, and V. Bhargava, "A general method for calculating error probabilities over fading channels," Communications, IEEE Transactions on, vol. 53, no. 5, pp. 841-852, 2005.

[9] M. Abramowitz and I. A. Stegun, Handbook of Mathematical Functions with Formulas, Graphs, and Mathematical Tables, 10th ed. Washington D.C.: National Bureau of Standards, Applied Mathematics Series - 55, 1972.

[10] J. G. Proakis, Digital Communications, 4th ed. McGraw-Hill International Edition, 2001.

[11] A. Goldsmith, Wireless Communications. Cambridge University Press, 2005.

[12] B. Sklar, Digital Communications: Fundamentals and Applications, 2nd ed. Prentice Hall, 2008.

[13] M. Nakagami, "Statistical characters of short-wave fading," vol. 27, 1943, pp. 145-.

[14] M. K. Simon and M.-S. Alouini, Digital communication over fading channels, 2nd ed. A Wiley-Interscience publication, 2005.

[15] N. Nielsen, Theorie des Integrallogarithmus und verwandter Transzendenten, ser. Cornell University Library historical math monographs. B. G. Teubner, 1906.

[16] A. Erdélyi, W. Magnus, F. Oberhettinger, F. G. Tricomi, and research associates, Tables of integral transforms: Based, in part, on notes left by Harry Bateman, ser. California Institute of technology. Bateman Manuscript project. McGraw-Hill, 1954, vol. 1.

[17] W. Feller, An Introduction to Probability Theory and Its Applications, 2nd ed. John Wiley \& Sons, 1971, vol. 2.

[18] N. L. Johnson, S. Kotz, and N. Balakrishnan, Continuous Univariate Distributions, 2nd ed. John Wiley \& Sons, 1995, vol. 2.

[19] I. S. Gradshteyn and I. M. Ryzhik, Table of Integrals, Series, and Products, 6th ed. London: Academic Press, 2000.

[20] N. W. Mc Lachlan, P. Humbert, and L. Poli, Supplément au formulaire pour le calcul symbolique. Gauthier-Villars, 1950.

[21] G. H. Hardy, J. E. Littlewood, and G. Pólya, Inequalities, ser. Cambridge Mathematical Library. Cambridge University Press, 1952.

[22] N. Bleistein and R. A. Handelsman, Asymptotic Expansions of Integrals. New York: Dover Publications, 1986. 